\title{
Burden and risk factors of diabetes and hyperglycemia in India: findings from the Global Burden of Disease Study 2016
}

This article was published in the following Dove Press journal: Diabetes, Metabolic Syndrome and Obesity:Targets and Therapy

\author{
Jaya Prasad Tripathy ${ }^{1,2}$ \\ 'International Union Against \\ Tuberculosis and Lung Disease, The \\ Union South East Asia Office, New \\ Delhi, India; ${ }^{2}$ International Union \\ Against Tuberculosis and Lung \\ Disease, Paris, France
}

Background: Diabetes, hyperglycemia, and their complications are a growing problem in India. However, no comprehensive picture of this disease burden has yet been presented to date. Methods: I used aggregate data from the Global Burden of Disease 2016 to describe the burden of diabetes and its risk factors, chronic kidney disease (CKD) due to diabetes, and diseases caused by high fasting plasma glucose from 1990 to 2016 in India.

Results: Deaths due to diabetes accounted for 3.1\% (95\% uncertainty interval [UI]: 2.9-3.3) of all deaths in India in 2016, up from $0.98 \%$ (95\% UI: 0.87-1.1) of all deaths in 1990. Diabetes and hyperglycemia accounted for 27.5 million disability-adjusted life years in 2016, of which diabetes accounted for 10 million. Diabetes contributes to the causation of ischemic heart disease, stroke, CKD, peripheral artery disease, specific cancers, and tuberculosis via intermediate hyperglycemia. High body mass index, dietary factors (diet low in fruits, nuts and seeds, and whole grains), and tobacco use were the most important risk factors for diabetes.

Conclusion: Diabetes and CKD due to hyperglycemia pose a large and increasing burden in India. Urgent programs and policies are needed to reduce the identified risk factors for diabetes and its burden.

Keywords: diabetes, hyperglycemia, mortality, morbidity, chronic kidney disease, ischemic heart disease, tuberculosis

\section{Introduction}

Noncommunicable diseases (NCDs) are a major threat to global health responsible for $\sim 72 \%$ of all deaths in the year $2016 .{ }^{1}$ Diabetes mellitus (DM) is among the four major NCDs warranting immediate attention. International Diabetes Federation estimated that $\sim 425$ million people had DM in 2017 and this number is expected to rise to 629 million by 2045 . DM claimed $\sim 4$ million adult deaths in 2017 . More than $75 \%$ of subjects with DM live in low- and middle-income countries (LMICs). ${ }^{2}$ The actual extent and size of the burden attributable to DM is still unknown to us especially in data-deficient LMICs. Apart from causing death and disability directly, diabetes also affects populations though its dreaded complications, most notably cardiovascular and renal disease, requiring complex approaches to get a complete picture of the burden of the disease. This requires a sound national database for disease incidence, prevalence, morbidity, including hospitalization, and mortality, which is incipient and incomplete in most LMICs.

The Global Burden of Disease (GBD) study quantifies burden of diseases, injuries, and risk factors from 1990 to the present, allowing comparisons over time, across age
Correspondence: Jaya Prasad Tripathy International Union Against Tuberculosis and Lung Disease, The Union South Asia Office, C-6 Qutub Institutional Area, New Delhi-I10016, India Email ijay.doc@gmail.com 
groups, and among populations. GBD offers LMICs valuable information on their disease burden, which aid in informed decision-making. To make these results more accessible and interpretable, Institute for Health Metrics Evaluation has put large volumes of information into a suite of interactive data visualizations that allow people to make sense of it. ${ }^{3}$

India, considered as the "diabetes capital" of the world, is home to 74 million diabetics with a prevalence of $\sim 8.7 \%$ among the adult population. ${ }^{2}$ Over the past decade, the national health information systems have improved, and a series of surveys have been conducted to estimate the burden of NCDs and its risk factors. The GBD study has incorporated this information into their standardized burden estimation approach. According to the GBD study, burden of diabetes implies those due to uncomplicated diabetes and its microvascular complications. ${ }^{4}$ However, there are several other diseases for which diabetes is considered causal via intermediate hyperglycemia.

In this report, I present the burden of diabetes, burden of other diseases attributable to diabetes via high fasting plasma glucose (HFPG), burden of chronic kidney disease (CKD) due to DM, and the risk attribution of several factors to DM in India from the GBD study in 2016.

\section{Methods}

The Global Burden of Diseases, Injuries and Risk Factors Study 2016 covers 195 countries, 7 super regions, and 21 regions from 1990 to 2016. In total, 333 causes of diseases and injuries, 264 causes of deaths, and 84 risk factors were systematically analyzed. Details of the GBD methodology have been explained in previous publications. ${ }^{1,5-7}$ I generated the estimates presented here from the GBD Data Visualization Tool maintained at the Institute for Health Metrics and Evaluation. $^{3}$

The framework used in the assessment of the burden of HFPG and diabetes and the burden attributed to its risk factors has already been used in other similar publications elsewhere. ${ }^{4,8}$ In GBD study, diabetes is defined as uncomplicated diabetes with the following microvascular sequelae: retinopathy (leading to moderate low vision, severe low vision, and blindness), diabetic neuropathy, diabetic foot, and amputation resulting from diabetes. The GBD framework recognizes diabetes both as a disease (uncomplicated DM), with its microvascular complications as outcomes, and as one of the multiple causes of several other diseases, such as, ischemic heart disease, chronic kidney disease, stroke, tuberculosis, Alzheimer's disease, peripheral artery disease, and cancers. This latter expression of the causal role of diabetes is accounted via intermediate HFPG.

The GBD study used a theoretical minimum risk exposure level of 4.8-5.4 mmol/L or 86.4-97.2 mg/dL for HFPG. This level is lower than the current cutoff point for the diagnosis of diabetes $(7.0 \mathrm{mmol} / \mathrm{L}$ or $126 \mathrm{mg} / \mathrm{dL}){ }^{6}$ Evidence from previous literature shows that HFPG can increase the risk of ischemic heart disease, stroke, CKD, peripheral artery disease, Alzheimer's disease, tuberculosis, and certain cancers. Relative risks for the association of HFPG and these outcomes have been provided elsewhere. ${ }^{6}$

The GBD study also identified the risk factors of diabetes such as high body mass index (BMI), dietary factors (diet low in fruits, nuts and seeds, and whole grains), alcohol use, occupational carcinogens, diet high in processed meat, low physical activity and tobacco use. The fraction of the diabetes burden attributable to each was calculated as previously described. ${ }^{6}$

The current study reports disability-adjusted life years (DALYs), deaths, and prevalence to quantify the burden of the disease. I compared the burden estimates over a period of 1990-2016. I also report a 95\% uncertainty interval (UI) for each estimate in our analysis.

No approval by a research ethics committee was deemed necessary for this study, as it utilized only secondary databases which are publicly available.

\section{Results}

\section{Trend in the burden of diabetes in India}

The diabetes death rate per 100,000 population increased from 10.0 (95\% UI: 8.9-11.0) in 1990 to 23.1 (95\% UI: 21.4-24.5) in 2016. Deaths due to diabetes accounted for $3.1 \%$ (95\% UI: $2.9-3.3)$ of all deaths in the region in 2016 , up from $0.98 \%$ (95\% UI: $0.87-1.1$ ) of all deaths in 1990 . The number of deaths due to DM also has nearly tripled during the same period.

The rate of DALYs from diabetes per 100,000 population increased from 440.1 (95\% UI: 374.5-523.6) in 1990 to 791.5 (95\% UI: $682.1-925.8$ ) in 2016 , an $80 \%$ increase. In terms of absolute numbers, there was a $174 \%$ increase in DALYs during the same period from 3.8 (3.2-4.5) million to $10.4(9.0-12.2)$ million DALYs. DALYs due to diabetes accounted for $2.2 \%$ (95\% UI: $2.0-2.4)$ of all DALYs in the country in 2016 , up from $0.7 \%$ (95\% UI: $0.6-0.8)$ in 1990 , a $215 \%$ jump.

The overall prevalence of DM rose $66 \%$ during the period of 1990-2016, from 3\% (95\% UI: $2.7-3.3)$ to 5\% (95\% UI: 4.5-5.5), whereas the age-standardized prevalence of diabetes 
increased by $30 \%$ during the same period, from $4.6 \%(95 \%$ UI: $4.1-5.0)$ to $6.0 \%$ (95\% UI: $5.4-6.5$ ).

\section{Burden due to combined diabetes and hyperglycemia}

Overall, diabetes and hyperglycemia accounted for 27.5 million DALYs in 2016. Of them, 37.8\% ( 10 million DALYs) arose directly from sufferings due to living with uncomplicated diabetes or from direct diabetes microvascular complications; $29.1 \%$ were from ischemic heart disease; $15.8 \%$ from CKD; $10.8 \%$ from stroke; $4.9 \%$ from tuberculosis; $0.8 \%$ from specific cancers; $0.6 \%$ from Alzheimer's disease; and $0.08 \%$ from peripheral artery disease as a result of intermediate hyperglycemia (Figure 1).

\section{HFPG: attributable DALYs}

The population attributable fraction of HFPG was $6.0 \%(95 \%$ UI: 5.0-7.1) of all DALYs 2016, which increased from $2.3 \%$ (95\% UI: 1.9-2.7) in 1990. HFPG was responsible for 56.6\% (95\% UI: 49.8-63.5) of all DALYs due to CKD, 24.4\% (95\% UI: 21.4-27.5) of all DALYs due to peripheral artery disease, 19.8\% (95\% UI: 12.6-29.8) of all DALYs due to ischemic heart disease, $18.2 \%$ (95\% UI: 12.0-26.3) of all DALYs due to stroke, 9.2\% (95\% UI: 5.9-12.5) of all DALYs due to tuberculosis, $7.8 \%$ (95\% UI: $1.7-16.8$ ) of DALYs due to Alzheimer's disease, and 4.9-6.6\% of DALYs due to various cancers in 2016 (Figure 2).

\section{HFPG: attributable deaths}

The population attributable fraction of HFPG was $10.5 \%$ (95\% UI: 8.5-12.8) of deaths from all causes in 2016, which increased from 4.4\% (95\% UI: $3.6-5.4)$ in 1990. In 2016, HFPG was responsible for $64.4 \%$ (95\% UI: $56.5-71.7)$ of all CKD deaths, $22.9 \%$ (95\% UI: 19.6-26.3) of deaths were due to peripheral artery disease, $21.1 \%$ (95\% UI: $13.1-32.1)$ deaths due to ischemic heart disease, $18.7 \%$ (95\% UI 12.427.3) deaths due to stroke, $9.8 \%$ (95\% UI: 6.2-13.8) deaths due to tuberculosis, $7.8 \%$ (95\% UI 1.7-16.7) deaths due to Alzheimer's disease, and 5.6-7.2\% deaths due to various cancers (Figure 3).

\section{Risk factors of diabetes}

Table 1 shows the contribution, in DALYs and deaths, of each diabetes risk factor. High BMI, dietary factors (diet low in fruits, nuts and seeds, and whole grains), and tobacco use were found to be the most important risk factors for diabetes. Figure 4 shows the relative size of the burden (in DALYs) due to HFPG, in relation to that of other risk factors. It shows that HFPG was the fifth leading cause of overall DALYs in India (Figure 4).

\section{Burden due to diabetes-related CKD}

Between 1990 and 2016, CKD death rates per 100,000 population from diabetes increased by 53\%, from 4.81 (95\% UI: $4.1-5.63)$ to 7.36 (95\% UI: 6.39-8.44). In 2016, 2,883,185 individuals (95\% UI: 2,516,507-3,288,409) died of CKD caused by diabetes in India. Among the total number of CKD deaths caused by diabetes, $63 \%$ occurred among males and $\sim 88 \%$ occurred among elderly aged more than 50 years.

The burden of CKD caused by diabetes per 100,000 population increased by $40 \%$, from 156.5 DALYs (95\% UI: 134-182) in 1990 to 219 DALYs (95\% UI: 191-250) in 2016

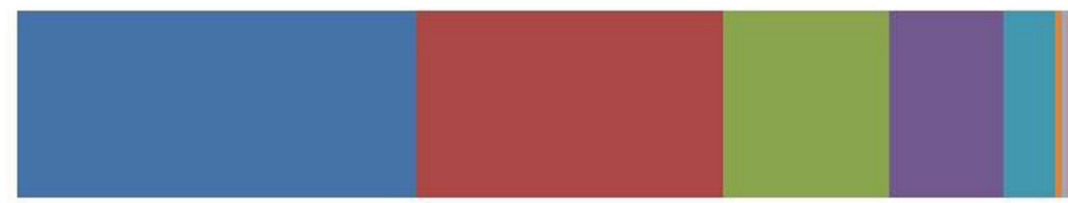

0 5
10
15

DALYs in millions
20

20

0

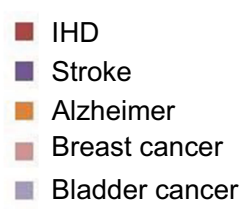

Figure I Distribution of DALYs attributable to high fasting plasma glucose, India, 2016.

Abbreviations: CKD, chronic kidney disease; DALYs, disability-adjusted life years; IHD, ischemic heart disease; TB, tuberculosis. 


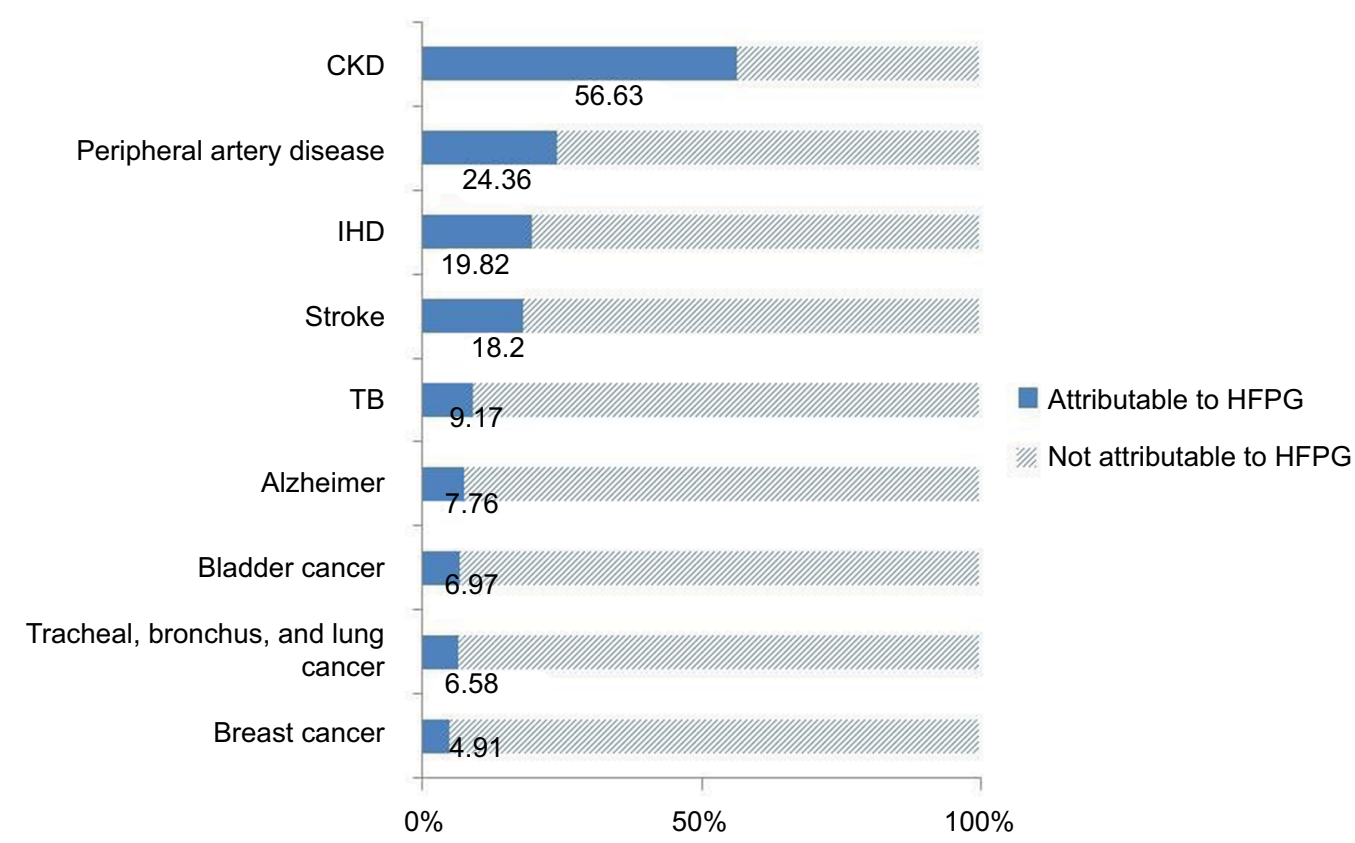

Figure 2 Proportion of DALYs due to diseases attributable to HFPG, India, 2016.

Abbreviations: CKD, chronic kidney disease; DALYs, disability-adjusted life years; HFPG, high fasting plasma glucose; IHD, ischemic heart disease; TB, tuberculosis

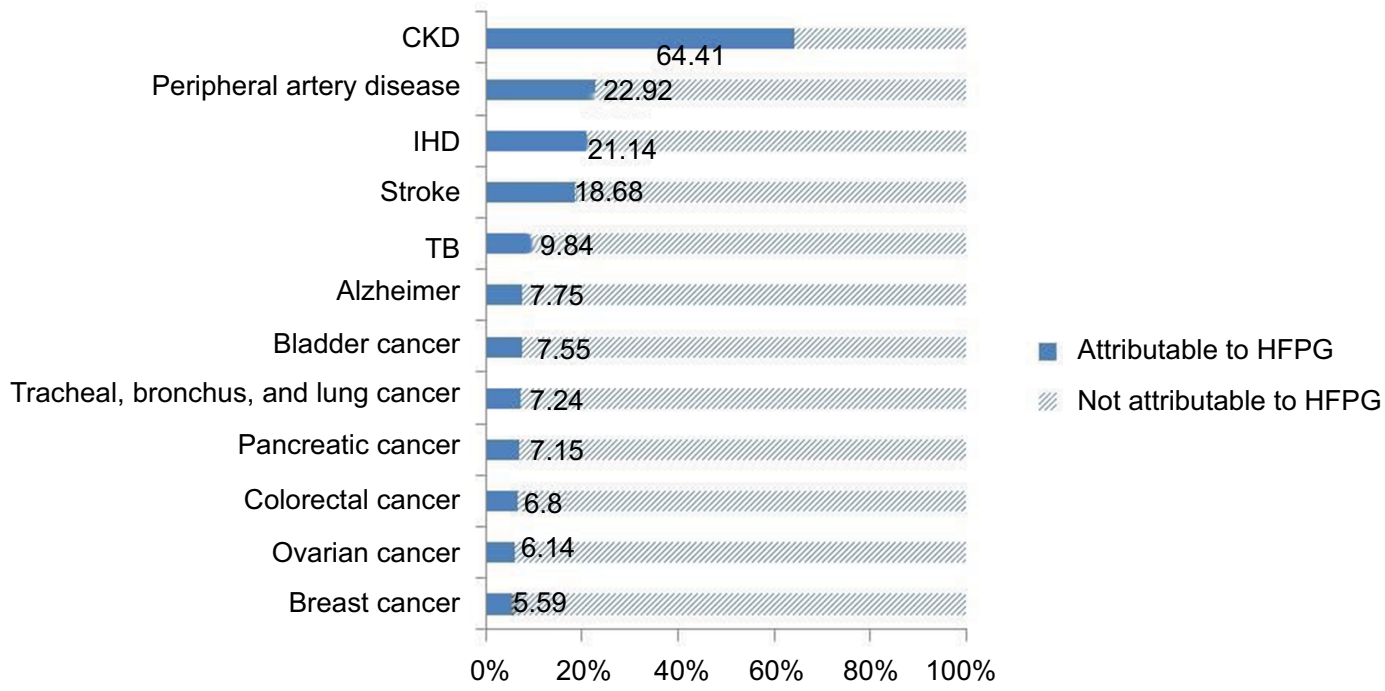

Figure 3 Proportion of deaths due to diseases attributable to HFPG, India, 2016.

Abbreviations: CKD, chronic kidney disease; HFPG, high fasting plasma glucose; IHD, ischemic heart disease; TB, tuberculosis.

Table I Risk factor attribution of DALYs and deaths due to diabetes resulting from different risk factors in India, 2015

\begin{tabular}{|c|c|c|c|c|c|c|}
\hline \multirow[t]{2}{*}{ Risk factors } & \multicolumn{3}{|c|}{ Risk factor attribution (DALYs) } & \multicolumn{3}{|c|}{ Risk factor attribution (deaths) } \\
\hline & $\%$ & 95\% UI & & $\%$ & 95\% UI & \\
\hline High fasting plasma glucose & 100 & & & 100 & & \\
\hline Diet high in processed meat & 0.16 & 0.00066 & 0.74 & 0.11 & 0.00047 & 0.55 \\
\hline Alcohol use & 1.02 & -0.34 & 2.57 & 0.55 & -0.67 & 1.93 \\
\hline Low physical activity & 2.24 & 0.51 & 4.19 & 2.54 & 0.57 & 4.71 \\
\hline Occupational carcinogens & 3.33 & 1.23 & 5.25 & 2.06 & 0.77 & 3.24 \\
\hline Diet low in whole grains & 5.54 & 2.02 & 11.07 & 4.54 & 1.65 & 9.17 \\
\hline Tobacco use & 10.72 & 6.07 & 15.43 & 11.07 & 6.31 & 15.89 \\
\hline Diet low in nuts and seeds & 10.99 & 5.34 & 17.47 & 8.73 & 4.2 & 13.98 \\
\hline Diet low in fruits & 12.06 & 2.67 & 21.98 & 9.69 & 2.12 & 17.88 \\
\hline High BMI & 35.96 & 22.58 & 49.18 & 24.77 & 14.4 & 36.02 \\
\hline
\end{tabular}

Abbreviations: BMI, body mass index; DALYs, disability-adjusted life years; UI, uncertainty interval. 


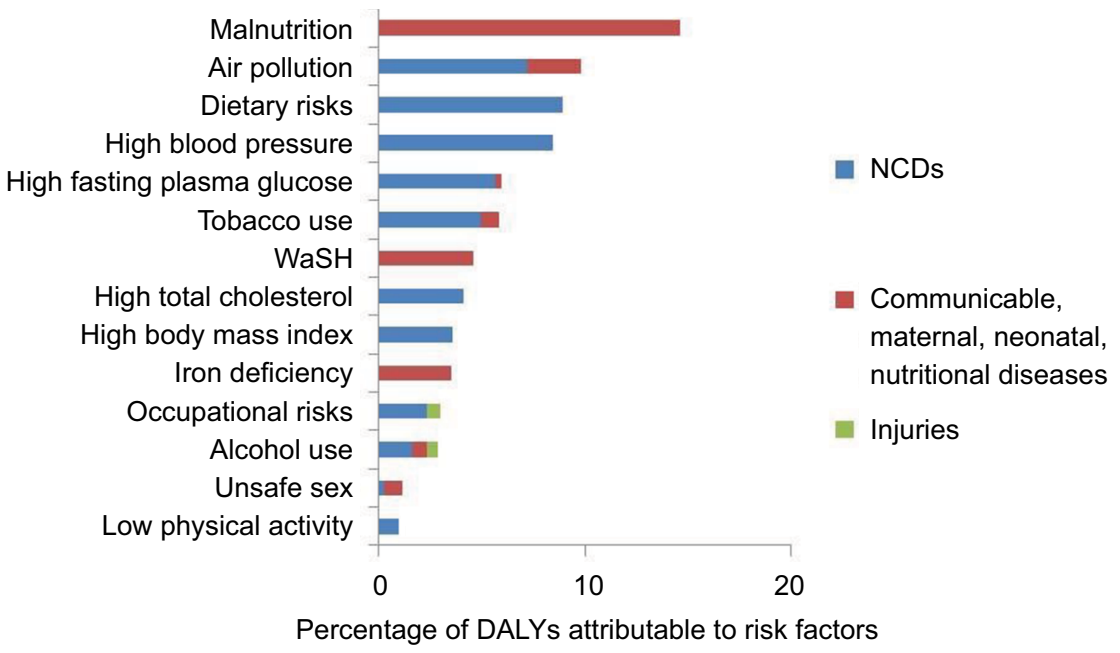

Figure 4 Relative burden of high fasting plasma glucose within the context of all risk factors identified by the GBD 2016 in India. Abbreviations: DALYs, disability-adjusted life years; GBD, Global Burden of Disease; NCDs, noncommunicable diseases; WaSH, water, sanitation and hygiene.

for all ages. The total number of DALYs caused by diabetesrelated CKD increased by $115 \%$ from 1.35 (95\% UI: $1.2-1.6)$ million in 1990 to 2.9 (95\% UI: 2.5-3.3) million in 2016.

\section{Discussion}

In the GBD study, DALYs resulting from diabetes are the results of living with uncomplicated diabetes or suffering its traditional microvascular complications. In this study, besides that, I have explored the burden, which includes that attributable via other diseases such as ischemic heart disease, stroke, CKD, peripheral artery disease, specific cancers, and tuberculosis for which diabetes and intermediate hyperglycemia are considered causal. To the best of our knowledge, this is the first comprehensive evaluation of the large and growing burden of diabetes and HFPG in India. Similar studies in the Eastern Mediterranean Region and Brazil have raised alarms about the increasing burden of diabetes and hyperglycemia., ${ }^{4,8}$

The key findings of the study are as follows: 1) the number of deaths and DALYs due to diabetes have nearly tripled during the period 1990-2016;2) overall, diabetes and hyperglycemia accounted for 27.5 million DALYs in 2016 in India, of which diabetes per se accounts for 10 million DALYs; 3) diabetes significantly contributes to the causation of ischemic heart disease, stroke, CKD, peripheral artery disease, specific cancers, and tuberculosis via intermediate hyperglycemia; 4) high BMI, dietary factors (diet low in fruits, nuts and seeds, and whole grains), and tobacco use were found to be the most important risk factors for diabetes; and 5) among all risk factors, HFPG was the fifth leading cause of overall DALYs in India.
Besides the burden of uncomplicated diabetes and its microvascular complications, diabetes also contributes to 17.5 million of additional DALYs due to its causal role in other diseases via hyperglycemia. This calls for individual and community level clinical and public health interventions to control blood sugar.

The presence of several factors not traditionally considered as conferring risk for diabetes such as low consumption of whole grains and seeds and nuts emphasize the importance of incorporating new evidence into public health and clinical actions. The study reported that diet low in nuts and seeds is a risk factor for diabetes, which is also supported by a meta-analysis of randomized controlled dietary trials. ${ }^{9}$ I support the inclusion of nuts as a part of a healthy diet for the management of glycemia in individuals with type 2 diabetes, although affordability is a concern in a country like India, which needs to be tackled at a policy level.

The study also reported that low intake of fruits and whole grains is a risk factor for diabetes. Afshin et al in a comparative risk assessment analysis also highlighted the role of dietary risk factors especially fruits and whole grains on diabetes mortality in the Middle East countries. ${ }^{10}$ However, consumption of whole grains and fruits and vegetables has decreased substantially in the region in the past two decades due to nutritional transition. This has been replaced by refined grains such as white bread, rice, and biscuits and diet rich in fat and sugar. ${ }^{11,12}$

High burden of diabetes in India is often a combination of dietary, behavioral, and metabolic risk factors. This study showed that high BMI is the main risk factor for diabetes similar to the findings in other studies. ${ }^{13,14}$ In recent decades, 
the prevalence of obesity has increased in the country and is predicted to increase further in the coming years in the absence of effective interventions. According to the recent estimates in the ICMR-INDIAB-3 study, obesity was prevalent among one-quarter of the respondents aged 20 years and older. ${ }^{15}$ Population-based public health strategies need to be implemented in various settings with a focus on health promotion and healthy lifestyle in order to tackle obesity.

The study reported high burden of CKD due to diabetes in terms of deaths and DALYs in India. With rising prevalence of disease risk factors such as diabetes and hypertension, prevalence of CKD is expected to rise. CKD is associated with enormous economic costs and premature mortality, which makes them a key target population to address. Taking cognizance of this problem, Government of India has rolled out the National Dialysis Program in 2016 to provide free dialysis to the poor. However, significant efforts are required toward prevention, screening, and early diagnosis of CKD.

There were few limitations in this study. First, despite the comprehensive nature of the GBD data set, these are model-based estimates with inherent limitations due to the model assumptions and the limited data used to build the model. Second, I did not differentiate subtypes of diabetes. However, the low prevalence of type 1 diabetes means that the results apply mainly to type 2 diabetes. Third, I did not examine the subnational/state-wise burden within the country, despite wide variations in risk factors and prevalence of conditions in different states. Fourth, it has been shown that HFPG increases the risk of other conditions such as some cancers, chronic liver disease, cataracts, periodontitis, pneumonia, and other infectious diseases (in addition to tuberculosis); ${ }^{16}$ thus, our attributable burden for HFPG might be underestimated.

\section{Conclusion}

The study findings show that diabetes causes a major burden in India directly and via other diseases, which are linked to intermediate hyperglycemia. The study provides necessary baseline information on the burden and risk profile of the disease to base their future diabetes reduction programs and enable them to monitor success of the program. Programs and policies are urgently needed to increase awareness about the disease, reduce the risk factors of diabetes, and improve the diagnosis and control of diabetes. This will reduce not only the burden of diabetes but also the burden of other diseases linked to this disease.

\section{Acknowledgments}

The author thanks the Department for International Development, UK, for funding this open access publication and for funding the Global Operational Research Fellowship Programme at the International Union Against Tuberculosis and Lung Disease, Paris, France, in which the author works as a Senior Operational Research Fellow. The author also acknowledges the GBD Project collaborators for putting data in the public domain and allowing relevant analysis and interpretations to aid decision-making.

\section{Disclosure}

The author reports no conflicts of interest in this work.

\section{References}

1. GBD 2016 Causes of Death Collaborators. Global, regional, and national age-sex specific mortality for 264 causes of death, 1980-2016: a systematic analysis for the Global Burden of Disease Study 2016. Lancet. 2017;390(10100):1151-1210.

2. International Diabetes Federation. IDF Diabetes Atlas - 8th edition. Available from: http://www.diabetesatlas.org. Accessed June 13, 2018.

3. Institute for Health Metrics and Evaluation. GBD Compare / Viz Hub; 2017. Available from: https://vizhub.healthdata.org/gbd-compare/. Accessed November 13, 2017.

4. Moradi-Lakeh M, Forouzanfar MH, El Bcheraoui C, et al. High fasting plasma glucose, diabetes, and its risk factors in the Eastern Mediterranean Region, 1990-2013: findings from the Global Burden of Disease Study 2013. Diabetes Care. 2017;40(1):22-29.

5. GBD 2016 DALYs and HALE Collaborators. Global, regional, and national disability-adjusted life-years (DALYs) for 333 diseases and injuries and healthy life expectancy (HALE) for 195 countries and territories, 1990-2016: a systematic analysis for the Global Burden of Disease Study 2016. Lancet. 2017;390(10100):1260-1344.

6. GBD 2016 Risk Factors Collaborators. Global, regional, and national comparative risk assessment of 84 behavioural, environmental and occupational, and metabolic risks or clusters of risks, 1990-2016: a systematic analysis for the Global Burden of Disease Study 2016. Lancet. 2017;390(10100):1345-1422.

7. GBD 2016 Disease and Injury Incidence and Prevalence Collaborators. Global, regional, and national incidence, prevalence, and years lived with disability for 328 diseases and injuries for 195 countries, 1990-2016: a systematic analysis for the Global Burden of Disease Study 2016. Lancet. 2017;390(10100):1211-1259.

8. Duncan BB, Schmidt MI, Cousin E, et al. The burden of diabetes and hyperglycemia in Brazil-past and present: findings from the Global Burden of Disease Study 2015. Diabetol Metab Syndr. 2017;9(1):18.

9. Viguiliouk E, Kendall CWC, Blanco Mejia S, et al. Effect of tree nuts on glycemic control in diabetes: a systematic review and metaanalysis of randomized controlled dietary trials. PLoS One. 2014; 9(7):e103376.

10. Afshin A, Micha R, Khatibzadeh S, et al. The impact of dietary habits and metabolic risk factors on cardiovascular and diabetes mortality in countries of the Middle East and North Africa in 2010: a comparative risk assessment analysis. BMJ Open. 2015;5(5):e006385.

11. Kearney J. Food consumption trends and drivers. Philos Trans $R$ Soc Lond B Biol Sci. 2010;365(1554):2793-2807.

12. Popkin BM, Adair LS, Ng SW. Global nutrition transition and the pandemic of obesity in developing countries. Nutr Rev. 2012;70(1): 3-21. 
13. Tripathy JP, Thakur JS, Jeet G, et al. Prevalence and risk factors of diabetes in a large community-based study in North India: results from a STEPS survey in Punjab, India. Diabetol Metab Syndr. 2017;9(1):8

14. Anjana RM, Deepa M, Pradeepa R, et al. Prevalence of diabetes and prediabetes in 15 states of India: results from the ICMR-INDIAB population-based cross-sectional study. Lancet Diabetes Endocrinol. 2017;5(8):585-596.
15. Pradeepa R, Anjana RM, Joshi SR, et al. Prevalence of generalized $\&$ abdominal obesity in urban \& rural India - the ICMR-INDIAB Study (Phase-I) [ICMR- NDIAB-3]. Indian J Med Res. 2015;142(2): $139-150$.

16. Rao Kondapally Seshasai S, Kaptoge S, Thompson A, et al. Diabetes mellitus, fasting glucose, and risk of cause-specific death. $N$ Engl J Med. 2011;364(9):829-841.
Diabetes, Metabolic Syndrome and Obesity: Targets and Therapy is an international, peer-reviewed open-access journal committed to the rapid publication of the latest laboratory and clinical findings in the fields of diabetes, metabolic syndrome and obesity research. Original research, review, case reports, hypothesis formation, expert opinion and commentaries are all considered for publication. The manuscript management system is completely online and includes a very quick and fair peer-review system, which is all easy to use. Visit http://www.dovepress.com/testimonials.php to read real quotes from published authors.

Submit your manuscript here: https://www.dovepress.com/diabetes-metabolic-syndrome-and-obesity-targets-and-therapy-journal 